\title{
Pulsatile blood pressure component as predictor of mortality in hypertension: a meta-analysis of clinical trial control groups
}

\author{
Jerzy Gasowskia,b, Robert H. Fagard ${ }^{a}$, Jan A. Staessen ${ }^{a}$, Tomasz Grodzickib ${ }^{b}$, \\ Stuart Pocock ${ }^{c}$, Florent Boutitie ${ }^{\mathrm{d}}$, François Gueyffier ${ }^{\mathrm{d}}$ and \\ Jean-Pierre Boissel ${ }^{d}$, for the INDANA Project Collaborators
}

\begin{abstract}
Objective Although current guidelines rest exclusively on the measurement of systolic and diastolic blood pressures, the arterial pressure wave is more precisely described as consisting of a pulsatile (pulse pressure) and a steady (mean pressure) component. This study explored the independent roles of pulse pressure and mean pressure as predictors of mortality in a wide range of patients with hypertension.
\end{abstract}

Design and methods This meta-analysis, based on individual patient data, has combined results from the control groups of seven randomized clinical trials conducted in patients with systolo-diastolic or isolated systolic hypertension. The relative hazard rates associated with pulse pressure and mean pressure were calculated using Cox's proportional hazard regression models with stratification for the seven trials and with adjustment for sex, age, smoking and the other pressure.

Results A $10 \mathrm{mmHg}$ wider pulse pressure at baseline, which corresponds to approximately one-half of its standard deviation, was independently associated with an increase in risk by $6 \%$ for total mortality $(P=0.001), 7 \%$ for cardiovascular mortality $(P=0.01)$, and $7 \%$ for fatal coronary accidents $(P=0.03)$.The corresponding increase in risk of fatal stroke was similar $(+6 \%, P=0.27)$ but there were too few strokes to reach statistical significance. In similar analyses, mean pressure was not identified as an independent predictor of these outcomes. Significant

\section{Introduction}

An elevated blood pressure acts in arteries as an injuring mechanical force, which may contribute to the development of atherosclerosis and to the increase in cardiovascular risk. The current guidelines for the detection and management of hypertension [1,2] rest on the measurement of the extreme values of blood pressure during the heart cycle, that is systolic and diastolic blood pressure. However, the arterial pressure wave is more precisely described as consisting of a pulsatile (pulse pressure) and a steady (mean pressure) component. Several observational studies showed that pulse pressure may be an independent predictor of cardiovascular risk interactions of pulse pressure or mean pressure with age suggested that the prognostic power of pulse pressure for fatal stroke was more important at higher age $(P=0.04)$, whereas the prognostic power of mean pressure for coronary mortality was greatest in the young $(P=0.01)$.

Conclusions In hypertensive patients pulse pressure, not mean pressure, is associated with an increased risk of fatal events. This appears to be true in a broad range of patients with hypertension. J Hypertens 20:145-151 () 2002 Lippincott Williams \& Wilkins.

Journal of Hypertension 2002, 20:145-151

Keywords: meta-analysis, pulse pressure, mean pressure, mortality, hypertension, clinical trial, randomized

${ }^{a}$ Hypertension and Cardiovascular Rehabilitation Unit, Department of Molecular and Cardiovascular Research, University of Leuven, Belgium; ${ }^{b}$ Department of Gerontology and Family Medicine, Jagiellonian University Medical College, Cracow, Poland; ' London School of Hygiene and Tropical Medicine, London, UK and ${ }^{\mathrm{d} D e p a r t m e n t}$ of Clinical Pharmacology, Claude Bernard University, Lyon, France.

Sponsorship: This project has been partially sponsored in the framework of the BIOMED 2 program (BMH4-CT98-3291). J.G. received a research fellowship from the Laboratories Schwartz Pharma (Boulogne-Billancourt, France). R.F. is holder of the Professor A. Amery Chair in Hypertension Research, founded by Merck Sharp and Dohme, Belgium.

Correspondence and request for reprints to Professor Robert H. Fagard, IGHypertensie, U.Z. Gasthuisberg, Herestraat 49, B-3000 Leuven, Belgium. Tel: +32/16/348707; fax +32/16/343766; e-mail: Robert.Fagard@uz.kuleuven.ac.be

Received 28 December 2000 Revised 7 September 2001 Accepted 10 September 2001

in the population [3-8], in hypertensive patients [9-13], in survivors of myocardial infarction [14], and in patients with left ventricular dysfunction [15]. A recently conducted analysis of the combined data from the EWPHE [16], Syst-Eur [17] and Syst-China [18] trials, showed that in elderly hypertensives, of whom most had isolated systolic hypertension, pulse pressure and not mean pressure predicted subsequent cardiovascular events [19]. However, it has not yet been fully studied whether this finding is consistent in hypertensives with wider age and blood pressure range. The collaboration within the framework of the INDANA project [20] allowed us to examine this question. 


\section{Methods}

In this overview, we included seven trials (Table 1). Six of these (EWPHE [16], HEP [21], MRC1 [22], MRC2 [23], SHEP [24], STOP [25]) had been incorporated in the INDANA database. The data of the Systolic Hypertension in Europe (Syst-Eur) Trial [17] and of the European Working Party on High Blood Pressure in the Elderly (EWPHE) trial [16] are held by the Study Co-ordinating Office at the Hypertension Unit of the University of Leuven, where this analysis was designed and conducted. Of the trials included in the INDANA database, we excluded the following: Multiple Risk Factors Intervention Trial [26] (MRFIT) and Hypertension Detection and Follow-up Program [27] because the control groups were not left untreated. The Australian Trial in Mild Hypertensives [28] was excluded because the final mortality survey was not carried out. The trials included tested different antihypertensive regimens. To test the predictive value of blood pressure components irrespective of treatment, we decided to include the data from the control groups only. Finally, only the mortality data were analysed, because in some trials such as EWPHE [16], non-fatal events were not recorded in patients who withdrew from double-blind treatment. Furthermore, fatal events are less easily misclassified than non-fatal ones and the definitions of the non-fatal events differed among several of the trials.

The outcomes studied included: total mortality, cardiovascular mortality, fatal stroke, and fatal coronary heart disease. The events have been defined as in the INDANA database [20]. Fatal coronary events included fatal myocardial infarction and sudden death (any death of unknown cause occurring within $24 \mathrm{~h}$ upon onset of symptoms). Cardiovascular mortality comprised fatal stroke, fatal coronary heart disease, fatal heart failure and other fatal cardiovascular events.

Database management and statistical analysis of the individual patient data from control groups were per- formed with SAS software (SAS Institute Inc, Cary, North Carolina, USA) [29]. First, after stratification for trial and adjustment for age and sex, we subdivided the distribution of systolic, diastolic, pulse pressure and mean pressure into thirds, and counted the incidence rates in those thirds. Logistic regression with pressure tertiles coded as single three-level categorical variables, was used to test for the linear trend. Then, pulse pressure and mean pressure were correlated with mortality using Cox's proportional hazard model. We report the relative hazard rates (RHR) for a $10 \mathrm{mmHg}$ wider pulse pressure or a $5 \mathrm{mmHg}$ higher mean pressure at baseline, which approximated to one-half of their respective standard deviations when the data were pooled. Stratification of the model accounted for the differences between the trials. The consistency of the results across studies was assessed by the application of a heterogeneity test [30]. Finally, to test the hypothesis that the risk attributable to pulse or mean pressure would be different at different ages, we tested the interaction between age and pulse pressure or mean pressure, respectively, after adjustment for confounders and the other pressure.

\section{Results}

\section{The general characteristics}

The control groups comprised 17239 patients. Their main characteristics are given in Table 1. Systolic and diastolic blood pressures averaged $( \pm$ SD) $170.4 \pm 18.3$ and $92.9 \pm 11.0 \mathrm{mmHg}$, respectively; pulse pressure averaged $( \pm S D) 77.5 \pm 20.8 \mathrm{mmHg}$ and mean pressure $118.7 \pm 9.9 \mathrm{mmHg}$. The coefficient of correlation between pulse pressure and mean pressure amounted to $0.17(P<0.001)$, and that between pulse pressure and systolic blood pressure was $0.85(P<0.001)$. Median age was 62.7 years and ranged from 26.3 to 97.0 years. Approximately one-quarter of all patients were younger than 50 years, particularly because of inclusion of the MRC1 trial.

Table 1 Characteristics of patients included in the control groups of the reviewed trials

\begin{tabular}{|c|c|c|c|c|c|c|c|}
\hline Trial & SHEP & Syst-Eur & MRC2 & HEP & MRC1 & EWPHE & STOP \\
\hline Main reference & 24 & 17 & 23 & 21 & 22 & 16 & 25 \\
\hline Mean age at entry (years) & 71.5 & 70.2 & 70.3 & 69.1 & 52.1 & 71.9 & 75.6 \\
\hline Systolic pressure at entry $(\mathrm{mmHg})$ & 170.1 & 173.9 & 184.4 & 196.5 & 161.6 & 182.4 & 195.3 \\
\hline Diastolic pressure at entry $(\mathrm{mmHg})$ & 76.7 & 85.5 & 90.8 & 97.4 & 98.3 & 100.5 & 101.7 \\
\hline Pulse pressure at entry $(\mathrm{mmHg})$ & 93.4 & 88.5 & 93.6 & 99.1 & 63.3 & 81.8 & 93.6 \\
\hline Mean pressure at entry $(\mathrm{mmHg})$ & 107.8 & 115.0 & 122.0 & 130.5 & 119.4 & 127.8 & 132.9 \\
\hline Number of patients in control group & 2371 & 2297 & 2213 & 465 & 8654 & 424 & 815 \\
\hline Women (\%) & 57 & 66 & 58 & 68 & 48 & 71 & 62 \\
\hline Smokers (\%) & 12.9 & 7.1 & 21.9 & 17 & 29.3 & 15.8 & 7.6 \\
\hline Median follow-up (years) & 4.4 & 2.0 & 6.0 & 3.5 & 5.3 & 4.4 & 2.1 \\
\hline All-cause deaths (rate per 1000 patient-years) & 23.7 & 25.2 & 24.8 & 33.7 & 5.9 & 76.8 & 35.4 \\
\hline Cardiovascular deaths (rate per 1000 patient-years) & 11.0 & 14.0 & 14.1 & 24.1 & 3.2 & 47.9 & 23.1 \\
\hline Fatal strokes (rate per 1000 patient-years) & 1.4 & 3.2 & 3.3 & 7.2 & 0.6 & 16.0 & 8.4 \\
\hline Fatal coronary heart diease (rate per 1000 patient-years) & 7.1 & 7.5 & 8.6 & 13.5 & 2.3 & 17.0 & 11.2 \\
\hline
\end{tabular}

The trials are in order of diastolic blood pressure at entry. 


\section{Analysis in tertiles of pressure components}

With stratification for trial and adjustment for age and sex, cardiovascular mortality increased from the lowest to the highest tertile of systolic pressure and pulse pressure (Fig. 1). For systolic pressure this trend was significant not only for cardiovascular mortality, but also for stroke mortality and all deaths. For pulse pressure, in addition to cardiovascular mortality, the trend was significant for fatal coronary heart disease and total mortality. The corresponding associations with diastolic pressure and mean pressure were not significant.

\section{Cox regression analysis}

In Cox regression with pulse pressure or mean pressure as the main explanatory variable, we stratified for trial and allowed for sex, age, smoking, and the alternative pressure component, i.e., the hazard rates for pulse pressure were adjusted for mean pressure and vice versa. In none of these analyses the hypothesis of homogeneity across the trials was rejected $\left(\chi_{6}{ }^{2} \leqslant 11.8\right.$, $P \geqslant 0.07$; Fig. 2 and Fig. 3). After adjustment for mean pressure and the other covariates, a $10 \mathrm{mmHg}$ wider pulse pressure was associated with a higher risk of total [RHR 1.06, 95\% confidence interval (CI) 1.03-1.10; $P=0.001]$, cardiovascular (RHR 1.07, 95\% CI 1.01$1.13 ; P=0.01$ ), and coronary (RHR 1.07, 95\% CI $1.01-1.15 ; P=0.03$ ) mortality (Fig. 2). However, this association was not statistically significant for stroke mortality. In similar analyses, mean pressure was not significantly associated with outcome (Fig. 3). When systolic and pulse pressure were included together in the models, neither of the two was significantly related to the risk of any of the studied end points.

\section{Cox regression analysis after exclusion of MRC1 trial}

Because of the younger age of the patients in the MRC1 trial, Cox regression analysis was also performed without the results from this trial. After adjustment for mean blood pressure and the other covariates, a $10 \mathrm{mmHg}$ wider pulse pressure was associated with a higher risk of total mortality (RHR 1.08, 95\% CI 1.031.13; $P<0.001)$ and of cardiovascular mortality (RHR $1.07,95 \%$ CI $1.01-1.14 ; P=0.01)$. The relative hazard rates were not significant for cause-specific mortality.

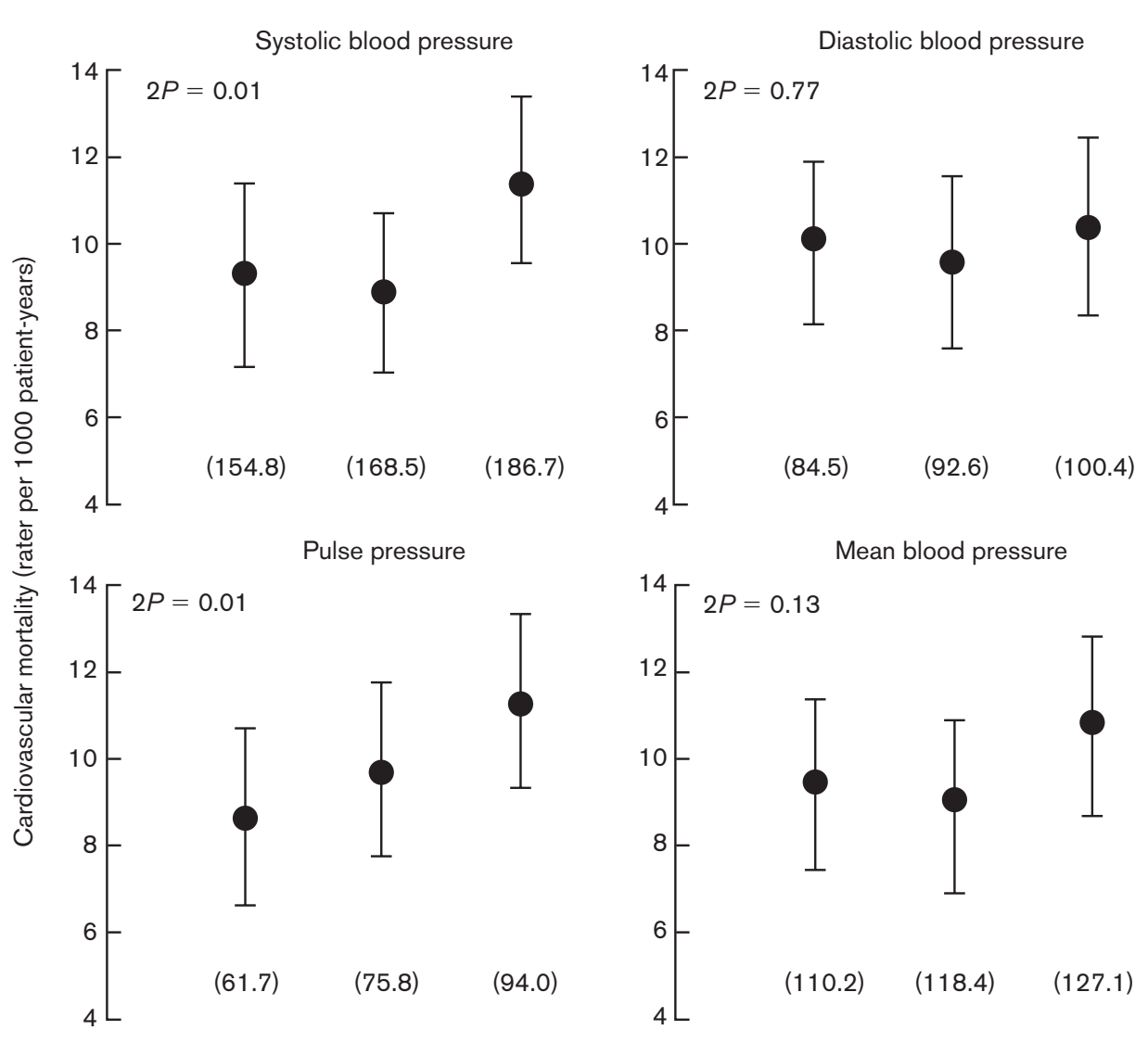

Rates ( \pm SEM) of fatal cardiovascular events in the control groups of seven trials, plotted in thirds of the respective pressure components after stratification by trial and adjustment for age and sex; numbers between brackets represent mean within-tertile BP values. $P$ values are for linear trends. 
Fig. 2

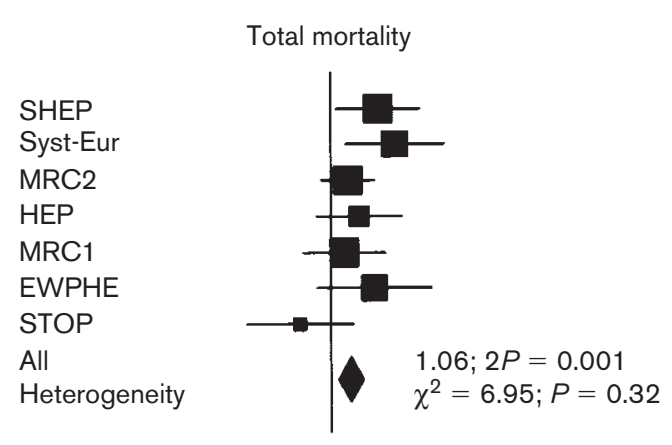

Cardiovascular mortality
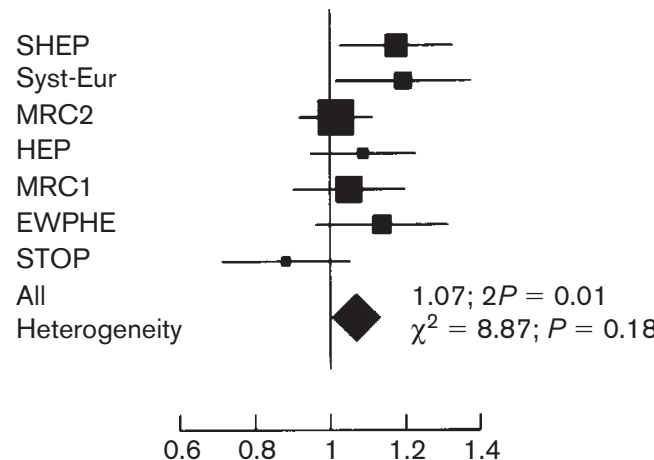

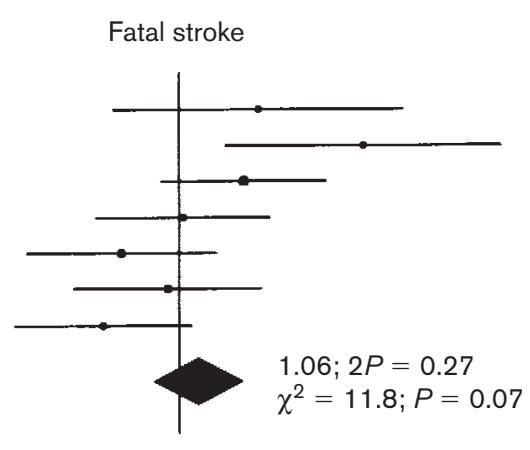

Fatal coronary events
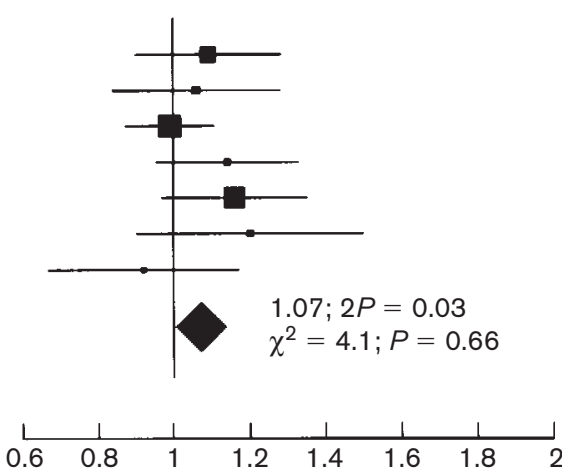

Relative hazard rate (95\% CL)

Relative hazard rates of fatal outcome, independently associated with a $10 \mathrm{mmHg}$ wider pulse pressure, adjusted for sex, age, smoking, and mean pressure, for each trial separately; the size of the squares corresponds to the numbers of events in each trial. Diamonds represent the $95 \%$ confidence intervals of the pooled estimates, after stratification by trial. Chi-square statistics for the heterogeneity of the data did not reach statistical significance.

The relationships between mean blood pressure and mortality were not significant, except for stroke mortality, which became significant upon exclusion of MRC1 patients from the analysis (RHR 1.10, 95\% CI 1.00$1.21 ; P=0.05)$.

The interaction between age and pressure components

The interactions between age and, respectively, pulse pressure and mean pressure, were not statistically significant for all-cause mortality and for cardiovascular mortality. However, the pulse pressure-age interaction reached significance for stroke mortality $(P=0.04)$. The RHR was 0.71 in the youngest tertile (age $\leqslant 56$ years) and was between 1.13 and 1.16 in the next three decades. The interaction between mean pressure and age was significant for fatal coronary heart disease $(P=0.01)$. The RHR amounted to 1.10 in the youngest tertile (age $\leqslant 56$ years), 1.08 in the middle tertile (ages $56-68$ years) and 0.97 at age $>68$ years.

\section{Discussion}

We found that in patients with hypertension a $10 \mathrm{mmHg}$ wider pulse pressure is independently asso- ciated with $6-7 \%$ increases in the risk of death from any cause or from cardiovascular complications. These risks were consistent throughout a large pool of patients enrolled as controls and assigned to placebo or no medication in seven randomized clinical trials. After adjustment for pulse pressure and confounders, mean blood pressure did not significantly add to the estimation of prognosis. There was no significant effect of age on these findings

With regard to cause-specific mortality, we observed a significant association of pulse pressure with fatal coronary heart disease but not with stroke mortality, whereas mean blood pressure did not have significant predictive power for these outcomes. However, when we analysed the possible influence of age, we observed a positive interaction between pulse pressure and age for stroke mortality, and a negative one between mean pressure and age for fatal coronary artery disease, meaning that the prognostic value of pulse pressure for stroke became more important at older age and that the risk of a higher mean arterial pressure for coronary heart disease was highest at younger age. 

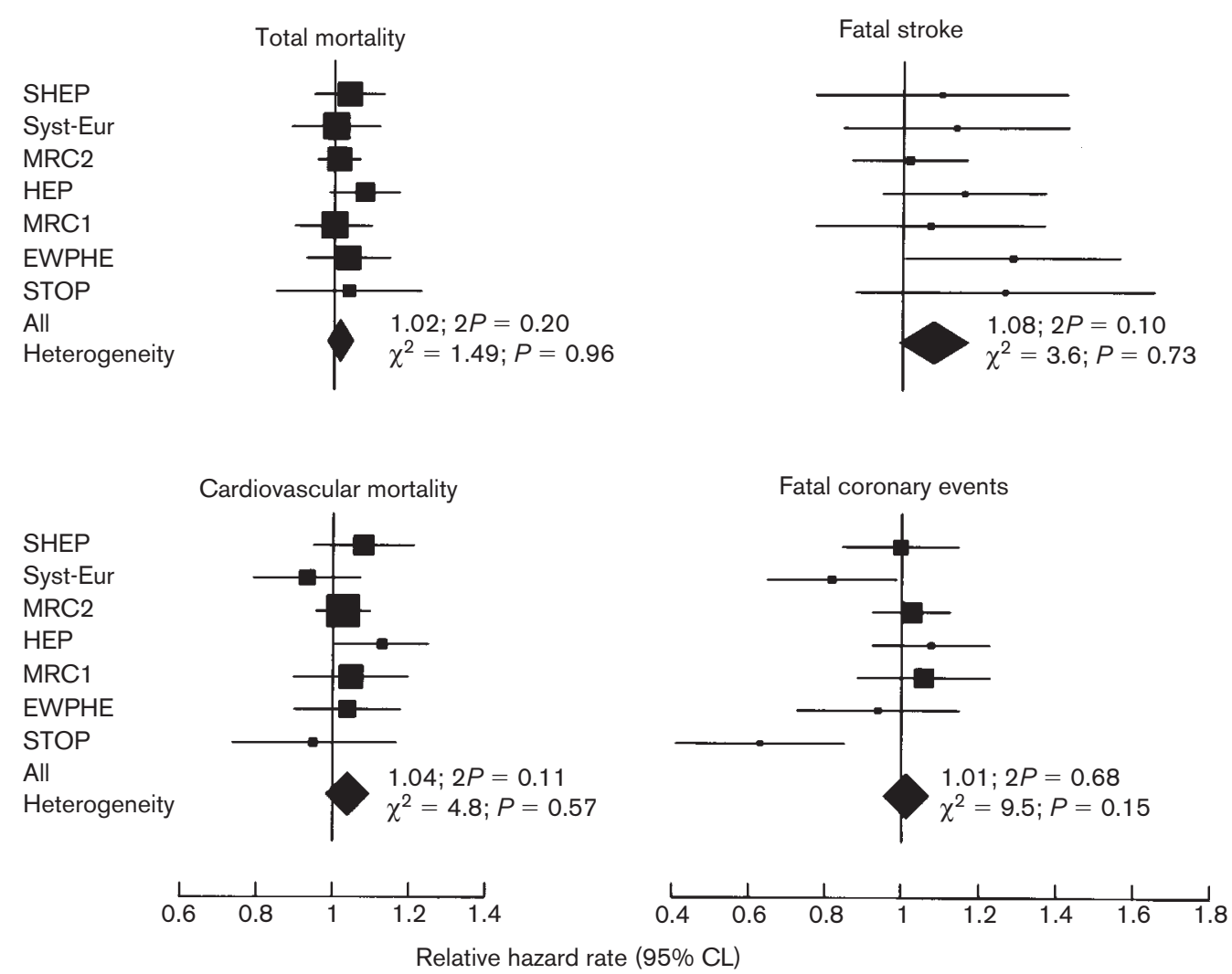

Relative hazard rates of fatal outcome, independently associated with a $5 \mathrm{mmHg}$ higher mean pressure, adjusted for sex, age, smoking, and pulse pressure, for each trial separately; the size of the squares corresponds to the numbers of events in each trial. Diamonds represent the $95 \%$ confidence intervals of the pooled estimates, after stratification by trial. Chi-square statistics for the heterogeneity of the data did not reach statistical significance.

The overall results on total mortality and on cardiovascular mortality were not affected by exclusion of the MRC1 trial, which included patients from young age up to the age of 65 years, whereas the minimal age was at least 60 years in the other trials. However, the predictive power of pulse pressure for coronary mortality was no longer significant, whereas mean blood pressure gained importance for the prediction of fatal stroke.

The present results confirm and expand the previously reported findings in elderly hypertensive patients that pulse pressure, rather than mean pressure, is the major risk factor of adverse cardiovascular events. Early reports from the Framingham Heart Study emphasized that systolic blood pressure was a better independent predictor of cardiovascular risk than diastolic pressure, particularly in subjects over the age of 50 [31,32]; a more recent analysis revealed that pulse pressure is a better predictor of the incidence of coronary artery disease than systolic or diastolic pressure [7]. The results from the MRFIT study indicated that patients were at particularly high risk when an elevated systolic blood pressure at baseline $(\geqslant 160 \mathrm{mmHg})$ was associated with a low diastolic pressure $(<70 \mathrm{mmHg})$ [33]. Darné et al. [3] were among the first to propose an independent role of pulse pressure as a risk factor, mainly for cardiac mortality, but only in women above 55 years of age. Several other groups confirmed the independent role of conventionally measured pulse pressure as a cardiovascular risk factor in the population [4-8], and in patients with hypertension [9-13], postmyocardial infarction [14] or left ventricular dysfunction [15]. It has also been observed that pulse pressure derived from $24 \mathrm{~h}$ ambulatory blood pressure monitoring is a potent predictor of cardiovascular outcome [11]. In a recently published meta-analysis, it was shown that in older patients with isolated elevation of systolic pressure, risk of death was positively associated with systolic blood pressure but inversely with diastolic pressure; consequently, the number of patients needed to be treated for 5 years to prevent one death was significantly lower in the high pulse pressure group [34]. 
Our estimation of the blood pressure-risk relationship could be influenced by the fact that the blood pressures were not freely observed but had to meet different entry criteria in each trial, and we advise caution when extrapolating these results to a more general population. However, the heterogeneity test confirmed the internal consistency of the results in a large collection of hypertensive patients enrolled in seven different trials. Moreover, in order to account for different trial designs, the analysis was stratified by trial.

In our Cox models, we tried to account for possible important confounders (sex, age and smoking, in addition to the other pressure). However, due to the lack of data for a large number of patients, we were unable to control for such factors as the level of total cholesterol or presence of diabetes mellitus.

Our analysis was not designed to check whether pulse pressure is a better predictor of mortality than systolic blood pressure. The role of pulse pressure as risk predictor was originally derived from an observation that in addition to the increase in risk of adverse events with rising systolic blood pressure, at any level of systolic blood pressure the lower the diastolic pressure, the higher the risk [19]. Only thereafter the question arose whether the pulsatile component would be more important than the steady component in the assessment of risk. Nevertheless, when we adjusted the pulse pressure for systolic pressure in the present analysis, neither of the two components remained a significant predictor of the mortality risk. This is what can be expected when so closely related variables are used in the same model. In fact, the strong interrelationship between systolic blood pressure and pulse pressure makes if difficult to distinguish between the role of the pulsatile component and of systolic blood pressure per se.

The present analysis was not designed to explain the possible mechanisms responsible for the better prediction of cardiovascular mortality by pulse pressure. However, it can be argued that hypertensive patients with increased pulse pressure are at increased risk of death because of more advanced arterial stiffness and atherosclerosis in various critical regions of the circulation, especially in coronary arteries and the arteries supplying the brain. In addition, increased pulsatile stretching may further damage the vasculature. For a diseased heart with atheromatous coronary arteries, a wide pulse pressure leads to less perfusion during diastole and a greater after-load, and thus higher wall tension due to stiffer conduit arteries. For the cerebral circulation, it means more mechanical force in systole, injuring vessels that lost their adaptive properties as a result of endothelial damage and stiffening, and a low perfusion during diastole.
In conclusion, the pooled results in control groups (without active treatment) of seven clinical trials, which included hypertensive patients with wide age and blood pressure range indicate that pulse pressure is an independent risk factor for overall and cardiovascular mortality. However, the present findings do not imply a causal relationship. In order to clarify the issue of whether or not lowering of pulse pressure would decrease cardiovascular morbidity and mortality, a specific, carefully designed clinical trial is needed.

\section{Acknowledgements}

The authors gratefully acknowledge the secretarial assistance of N. Ausseloos. They also greatly appreciate the collaboration of the other INDANA Steering Committee members (John Coope, Jeffrey Cutler, Tord Ekbom, Lawrence Friedman, Karla Kerlikowski, Mitchell Perry, Ronald Prineas and Eleanor Schron).

\section{References}

1 The sixth report of the Joint National Committee on prevention, detection, evaluation, and treatment of high blood pressure. Arch Intern Med 1997; 157:2413-2446. [Published erratum appears in Arch Intern Med 1998 Mar 23; 158 (6):573.]

2 Guidelines Subcommittee. 1999 World Health Organization - International Society of Hypertension Guidelines for the Management of Hypertension. J Hypertens 1999; 17:151-183.

3 Darné B, Girerd X, Safar M, Cambien F, Guize L. Pulsatile versus steady component of blood pressure: a cross-sectional and prospective analysis on cardiovascular mortality. Hypertension 1989; 13:392-400.

4 Benetos A, Safar M, Rudnichi A, Smulyan H, Richard JL, Ducimetiere P, et al. Pulse pressure: a predictor of long-term cardiovascular mortality in a French male population. Hypertension 1997; 30:1410-1415.

5 Benetos A, Rudnichi A, Safar M, Guize L. Pulse pressure and cardiovascular mortality in normotensive and hypertensive subjects. Hypertension 1998; 32:560-564.

6 Chae CU, Pfeffer MA, Glynn RJ, Mitchell GF, Taylor JO, Hennekens CH. Increased pulse pressure and risk of heart failure in the elderly. JAMA 1999; 281:634-639.

7 Franklin SS, Khan SA, Wong ND, Larson MG, Levy D. Is pulse pressure useful in predicting coronary heart disease? The Framingham Heart Study. Circulation 1999; 100:354-360.

8 Antikainen $\mathrm{RL}$, Jousilathi $\mathrm{P}$, Vanhanen $\mathrm{H}$, Tuomilehto J. Excess mortality associated with pulse pressure among middle-aged men and women is explained by high systolic pressure. J Hypertens 2000; 18:417-423.

9 Madhavan S, Ooi WL, Cohen H, Alderman MH. Relation of pulse pressure and blood pressure reduction to the incidence of myocardial infarction. Hypertension 1994; 23:395-401.

10 Fang J, Madhavan S, Cohen H, Alderman MH. Measures of blood pressure and myocardial infarction in treated hypertensive patients. J Hypertens 1995; 13: 413-419.

11 Verdecchia P, Schillaci G, Borgioni C, Ciucci A, Pede S, Porcellati C. Ambulatory pulse pressure: a potent predictor of total cardiovascular risk in hypertension. Hypertension 1998; 32:983-988.

12 Domanski MJ, Davis BR, Pfeffer MA, Kastantin M, Mitchell GF. Isolated systolic hypertension: prognostic information provided by pulse pressure. Hypertension 1999; 34:375-380.

13 Millar JA, Lever AF, Burke V. Pulse pressure as a risk factor for cardiovascular events in the MRC Mild Hypertension Trial. $J$ Hypertens 1999; 17:1065-1072.

14 Mitchell GF, Moyè LA, Braunwald E, Rouleau JL, Bernstein V, Geltman $\mathrm{EM}$, et al. Sphygmomanometrically determined pulse pressure is a powerful independent predictor of recurrent events after myocardial infarction in patients with impaired left ventricular function. Circulation 1997; 96:4254-4260

15 Domanski MJ, Mitchell GF, Norman JE, Exner DV, Pitt B, Pfeffer MA. Independent prognostic information provided by sphygmomanometrically determined pulse pressure and mean arterial pressure in patients with left ventricular dysfunction. J Am Coll Cardiol 1999; 33:951-958.

16 Amery A, Birkenhäger W, Brixko $P$, Bulpitt $C$, Clement $D$, Deruyttere $M$, 
et al. Mortality and morbidity results from the European Working Party on High Blood Pressure in the Elderly trial. Lancet 1985; i:1349-1354.

17 Staessen JA, Fagard R, Thijs L, Celis H, Arabidze GG, Birkenhäger WH, et al. Randomised double-blind comparison of placebo and active treatment for older patients with isolated systolic hypertension. Lancet 1997; 350:757-764. [Correction published in Lancet 1997; 350:1636.]

18 Liu L, Wang J, Gong L, Liu G, Staessen J. Comparison of active treatment and placebo in older Chinese patients with isolated systolic hypertension. J Hypertens 1998; 16:1823-1829.

19 Blacher J, Staessen JA, Girerd X, Gasowski J, Thijs L, Liu L, et al. Pulse pressure - not mean pressure - determines cardiovascular risk in older hypertensive patients. Arch Intern Med 2000; 160:1085-1089.

20 Gueyffier F, Boutitie F, Boissel JP, Coope J, Cutler J, Ekbom T, et al. INDANA: a meta-analysis on individual patient data in hypertension. Protocol and preliminary results. Thérapie 1995; 50:353-362.

21 Coope J, Warrender TS. Randomised trial of treatment of hypertension in elderly patients in primary care. BMJ 1986; 293:1145-1151.

22 Medical Research Council Working Party. MRC trial of treatment of mild hypertension: principal results. BMJ 1985; 291:97-104.

23 MRC Working Party. Medical Research Council trial of treatment of hypertension in older adults: principal results. BMJ 1992; 304:405-412.

24 SHEP Cooperative Research Group. Prevention of stroke by antihypertensive drug treatment in older persons with isolated systolic hypertension. Final results of the Systolic Hypertension in the Elderly Program (SHEP). JAMA 1991; 265:3255-3264.

25 Dahlöf B, Lindholm LH, Hansson L, Scherstén B, Ekbom T, Wester PO. Morbidity and mortality in the Swedish Trial in Old Patients with Hypertension (STOP-Hypertension). Lancet 1991; 338:1281-1285.

26 Multiple Risk Factor Intervention Trial Research Group. Multiple Risk Factor Intervention Trial: risk factor changes and mortality results. JAMA 1982; 248:1465-1477.

27 Davis BR, Ford CE, Remington RD, Stamler R, Hawkins CM. The Hypertension Detection and Follow-up Program design, methods, and baseline characteristics and blood pressure response of the study population. Prog Cardiovasc Dis 1986; 29 (suppl. 1):11-28.

28 Management Committee. The Australian Therapeutic Trial in Mild Hypertension. Lancet 1980; i:1261-1267.

29 SAS Institute Inc. SAS/STAT Software: Changes and Enhancements Through Release 6.11. Cary, North Carolina: SAS Institute Inc; 1996:807-884.

30 Armitage P, Berry G. Statistical methods in medical research. 4th ed. Cambridge: Blackwell Science; 1994.

31 Kannel WB, Gordon T, Schwartz MJ. Systolic versus diastolic blood pressure and risk of coronary heart disease. The Framingham Study. Am J Cardiol 1971; 27:335-346.

32 Kannel WB, Dawber TR, Sorlie P, Wolf PA. Components of blood pressure and risk of atherothrombotic brain infarction: the Framingham Study. Stroke 1976; 7:327-331.

33 Neaton J, Wentworth D, for MRFITRG. Serum cholesterol, blood pressure, cigarette smoking, and death from coronary heart disease. Overall findings and differences by age for 316099 white men. Arch Intern Med 1992; 152:56-64.

34 Staessen JA, Gasowski J, Wang JG, Thijs L, Den Hond E, Boissel J-P, et al. Risk of untreated and treated isolated systolic hypertension in the elderly: meta-analysis of outcome trials. Lancet 2000; 355:865-872. 